\title{
X-RAY BACKGROUND FLUCTUATIONS FROM DISCRETE SOURCES WITH A VARYING DENSITY CONTRAST
}

\author{
Honguang Bi \\ Max-Planck Institut für Astrophysik \\ Garching \\ FRG \\ P. Mészáros \\ Department of Astronomy \\ Pennsylvania State University \\ University Park, PA USA
}

\author{
A. Mészáros \\ Dept. of Astronomy and Astrophysics \\ Charles University \\ Prague, Czechoslovakia
}

\section{INTRODUCTION}

In two previous publications (Mészáros and Mészáros 1988 "Paper I"; Bagoly, Mészáros, and Mészáros 1988 "Paper II"), we have studied the fluctuations of the X-ray background (XRB) expected if the XRB is produced by discrete sources distributed as galaxies. The distribution of matter was assumed to follow the large-scale structure in the form of spherical clusters (superclusters) or spherical voids. In Paper I the density contrast of XRB sources inside and outside structures was taken to be a step function of constant height, whereas in Paper II we introduced arbitrary density contrasts (independent of redshift) and allowed for a redshift evolution of the luminosity of the sources. This led to predicted angular fluctuations of the XRB, which, when we compared them with the HEAO-1 observational limit (Shafer 1983), allowed us to set limits on the type and density of structures.

In the present paper we discuss the XRB angular fluctuation calculated for the case of a redshift-dependent density contrast, as would be expected in the course of the formation of the large-scale structures.

\section{Calculations}

We assume that the number of sources in a shell of thickness $d \chi$ (where $d \chi$ is the radial comoving coordinate, cf. Paper I and II) is

$$
d N(\chi)=d N_{\mathrm{u}}(\chi)+N_{\mathrm{c}}(\chi) d C(\chi),
$$

where $d N_{\mathrm{u}}(\chi)$ represents the sources in the uniform components and $N_{\mathrm{c}}(\chi)$ are the sources in one of the structures, of which there are $d C(\chi)$ in the shell. The density contrast between uniform and structured components is $\eta(\chi)$, so that

$$
d N_{\mathrm{u}}(\chi)=N_{c}(\chi) \frac{d C(\chi)}{\eta(\chi)}
$$

The luminosity evolution of individual sources is given by $J(\chi)=J_{0} \phi(\chi)$, for which we take the form indicated by quasar counts.

The total intensity of the XRB is $I_{\text {tot }}=I_{\mathrm{c}}+I_{\mathrm{u}}$, and the spatial fluctuations are given by

$$
\left[\frac{\delta I}{I}\right]^{2}=\frac{\left(\delta I_{\mathrm{c}}\right)^{2}+\left(\delta I_{\mathrm{u}}\right)^{2}}{I_{\mathrm{tot}}^{2}} .
$$

The distance-related fluctuations $\delta I_{\mathrm{c}}$ and $\delta I_{\mathrm{u}}$ are then calculated for various Friedmann models. 


\section{RESULTS}

We discuss results for some representative models, including $\Omega_{0}=1$ and $0.1, \phi(\chi)=1$, and $\phi(\chi)=e^{6 \tau}$, with $\tau=$ lookback time, and with constant comoving separation between structures, with present mean separation $L_{\mathrm{o}}=30 h^{-1} \mathrm{Mpc}$, and $H=h 100 \mathrm{~km} \mathrm{~s}^{-1} \mathrm{Mpc}^{-1}$. The integrations extend between $z_{1}=0.5$ and $z_{2}=4$. For simplicity we choose the total number of sources $d N(\chi)$ to be constant, which occurs if

$$
N_{\mathrm{c}}=N_{\mathrm{co}} \frac{\eta(\chi)}{1+\eta(\chi)},
$$

where $N_{\text {co }}=5,20$, or 100 . For $\eta(\chi)$ we took either a constant, a step function at $z_{f}$, or else

$$
\eta(\chi)=\left[0.99 \mathrm{e}^{-\left(\chi / \chi_{f}\right)^{n}}+0.01\right] 10 \text {, }
$$

where $z_{f}$ is the redshift at which the contrast becomes strong, and we took $z_{f}=1$.

Some of the numerical results for $\delta I / I$ are given in Table 1 , evaluated for a $5^{\circ} \times 5^{\circ}$ solid angle as in the HEAO-1 data (Shafer 1983). The values in Table 1 are the distance-related fluctuations. Other effects (cf. Paper II) will increase these slightly but not significantly.

\begin{tabular}{lrrcccc}
\multicolumn{7}{c}{ TABLE 1 } \\
\hline \hline$\Omega_{0}$ & $\phi(\chi)$ & $N_{\text {co }}$ & $\eta=10$ & $\begin{array}{c}\eta=10 \quad z<z_{f} \\
\eta=0.01 z>z_{f}\end{array}$ & $\begin{array}{c}\eta=\text { equ. } 5 \\
n=2\end{array}$ & $\begin{array}{c}\eta=\text { equ. } 5 \\
n=4\end{array}$ \\
\hline & & 5 & $2.50 \times 10^{-2}$ & $2.65 \times 10^{-2}$ & $2.41 \times 10^{-2}$ & $2.54 \times 10^{-2}$ \\
1 & 1 & 20 & $2.49 \times 10^{-2}$ & $2.63 \times 10^{-2}$ & $2.39 \times 10^{-2}$ & $2.51 \times 10^{-2}$ \\
& & 100 & $2.48 \times 10^{-2}$ & $2.60 \times 10^{-2}$ & $2.36 \times 10^{-2}$ & $2.49 \times 10^{-2}$ \\
& & 5 & $0.82 \times 10^{-2}$ & $1.07 \times 10^{-2}$ & $0.92 \times 10^{-2}$ & $1.07 \times 10^{-2}$ \\
1 & $\mathrm{e}^{6 \tau}$ & 20 & $0.82 \times 10^{-2}$ & $1.05 \times 10^{-2}$ & $0.91 \times 10^{-2}$ & $1.05 \times 10^{-2}$ \\
& & 100 & $0.82 \times 10^{-2}$ & $1.03 \times 10^{-2}$ & $0.90 \times 10^{-2}$ & $1.04 \times 10^{-2}$ \\
& & 5 & $0.83 \times 10^{-2}$ & $0.92 \times 10^{-2}$ & $0.79 \times 10^{-2}$ & $0.85 \times 10^{-2}$ \\
0.1 & 1 & 20 & $0.83 \times 10^{-2}$ & $0.91 \times 10^{-2}$ & $0.78 \times 10^{-2}$ & $0.84 \times 10^{-2}$ \\
& & 100 & $0.82 \times 10^{-2}$ & $0.90 \times 10^{-2}$ & $0.77 \times 10^{-2}$ & $0.83 \times 10^{-2}$ \\
& & 5 & $0.37 \times 10^{-2}$ & $0.51 \times 10^{-2}$ & $0.40 \times 10^{-2}$ & $0.48 \times 10^{-2}$ \\
0.1 & $\mathrm{e}^{6 \tau}$ & 20 & $0.37 \times 10^{-2}$ & $0.51 \times 10^{-2}$ & $0.39 \times 10^{-2}$ & $0.48 \times 10^{-2}$ \\
& & 100 & $0.37 \times 10^{-2}$ & $0.50 \times 10^{-2}$ & $0.39 \times 10^{-2}$ & $0.47 \times 10^{-2}$ \\
\hline
\end{tabular}

The observational upper limit is $\delta I I I \leqslant 2.3 \%$ and must be compared with the models of Table 1. For the non-evolving $\phi(\chi)=1$ models, the predicted fluctuations are too large in the case of $\Omega_{0}=1$ to be acceptable. On the other hand, for the luminosity evolution case, the predicted fluctuations do not impose any restrictions on $\Omega_{0}$. This in essence confirms our results of Paper II. The evolving contrast cases considered here (columns 2, 3, and 4) give similar results to the constant contrast case (column 1 and Paper II), because the fluctuations are dominated by the low redshift regions, where the contrast has saturated to a constant value. We conclude that an $\Omega_{0}=1$ universe, where the XRB is given by discrete sources, would require these to have a strong luminosity evolution of the type encountered in active galactic nuclei. A more detailed discussion is in preparation.

\section{REFERENCES}

Mészáros, A. and Mészáros, P. 1988, Ap. J., 327, 25.

Bagoly, Z., Mészáros, A., and Mészáros, P. 1988, Ap. J., 333, 54.

Shafer, R. 1983, Ph.D. thesis, University of Maryland (NASA Tech. Memo. 85029). 